MATEC Web of Conferences 40, 07013 (2016)

DOI: $10.1051 /$ matecconf/20164007013

C) Owned by the authors, published by EDP Sciences, 2016

\title{
A Method of Measuring AC HV Using HV Standard Capacitor
}

\author{
Jie CHEN ${ }^{1}$, Xian-hua HOU ${ }^{2}$, Li ZHOU ${ }^{1}$, Huan ZHENG ${ }^{2}$, Chen-ying LI' ${ }^{1}$, Zhong-hua $\mathrm{LI}^{2}$ \\ 1 Jiangsu Electric Power Research Institute, Nanjing Jiangsu \\ ${ }^{2}$ College of Electrical \& Electronic Engineering, Harbin University of Science and Technology
}

\begin{abstract}
According to the demand of new measuring AC high-voltage technology based on the development of power network, this paper proposes an $\mathrm{AC}$ high -voltage measuring system using high voltage standard capacitor based on the analysis of the existing AC high-voltage measuring technology, the working principle of the AC highvoltage measuring system is analyzed in detail, and the experimental research on the steady state characteristic and frequency characteristic of the AC high-voltage measuring system is made, at the same time, the possible errors are analyzed in detail. The AC high-voltage measuring system meets the technical requirements of high-voltage signal acquisition in the power system, at the same time, meets the requirements of $\mathrm{AC}$ high voltage measurement when the frequency is not more than $2500 \mathrm{~Hz}$, and has certain practical value.
\end{abstract}

\section{Introduction}

In recent years, operation voltage level of power grid is more and more high with the increase of power system capacity, on the one hand, the traditional AC high-voltage testing technology has been very difficult to meet the demand of power grid's development, on the other hand, the development of UHV power grid has an urgent need to develop new type of AC high-voltage measurement technology.

Existing AC high-voltage measurement techniques mainly include electromagnetic(PT) and capacitor or resistor divider sensing technology, In recent years, more advanced optical voltage sensing technology emerges. Some references also suggest that capacitor or resistor connect with hollow coil and capacitor or resistor connect with small current transformer[1]. The traditional electromagnetic voltage transformer technology is not suitable for the development of large capacity and intelligent power grid because of its difficulty in insulation, easy occurrence of ferromagnetic resonance. However, voltage transformer technology of resistor divider is limited by the power of resistance, only suitable for middle and low voltage power system. Voltage sensing technology of capacitive divider has many advantages such as electric field strength margin, high insulation etc .but high voltage capacitance is easy to be influenced by temperature, and capacitor discharge occurs in line short circuit[2]. Capacitance or resistance connect hollow coil or small current transformer has no active power consumption, but it can be influenced for the precision in a certain extent by environmental factors such as temperature, magnetic field etc. Optical voltage sensing technology mainly uses the physical characteristics of optical materials mainly includes electronic voltage transformer technology based on Pockels effect and inverse voltage effect, however, due to the high demand for materials and equipment, the crystal is easy to be affected by vibration, environmental temperature and other factors, the stability and reliability need to be further strengthened $[3,4]$.

This paper proposes an AC high-voltage measuring system based on high voltage standard capacitor. The standard capacitor and the current voltage conversion circuit are connected in series, the TVS tube plays a protective role in the operational amplifier, the high voltage standard capacitor introduced in this paper can eliminate the test error caused by the environment and other factors, which has the characteristics of no ferromagnetic resonance, high temperature stability, strong anti-interference ability, and high accuracy. The $\mathrm{AC}$ high-voltage measuring system can not only be applied to the power system of multiple voltage levels, but also can be used to form a test device for extracting high voltage signal in the laboratory.

\section{Basic principles of measuring system}

The AC high-voltage measuring system is made of high voltage standard capacitor, protective circuit and signal processing circuit, as shown in Figure 1. 


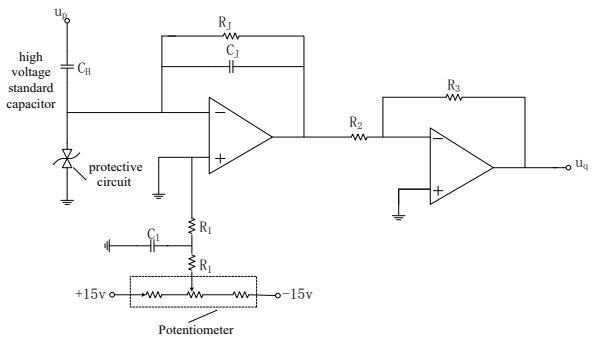

Figure 1. Diagram of measuring AC HV with high voltage standard capacitor

High voltage standard capacitor will change high voltage signal into capacitive current signal IC, as shown in equation (1):

$$
I_{\mathrm{C}}(t)=C_{\mathrm{H}} \frac{\mathrm{d} u_{\mathrm{p}}(t)}{\mathrm{d} t}
$$

Equation (1) shows that the capacitor current is proportional to the differential of high voltage signal. So it is necessary to carry out the integral to complete the reduction process, usually by inverse integrator in ideal integrator state, as shown in formula (2):

$$
u_{q}=-\frac{1}{C_{J}} \int I_{C}(t) d t=-\frac{C_{H}}{C_{J}} u_{p}(t)
$$

Where $\mathrm{C}_{\mathrm{J}}$ is an integral capacitor.

Equation (2) shows that the phase difference of the input voltage and the output voltage is $180^{\circ}$, so we can restore the phase of the output signal by inverter, as shown in equation (3):

$$
\frac{u_{q}}{u_{p}}=\frac{C_{H}}{C_{J}}
$$

But in the actual operational amplifier circuit, even if the maximum suppression of zero drift, zero drift still exist. So the integral capacitor is connected in parallel with a resistor as shown in Figure 1. resistance should be as far as possible to obtain bigger, so the phase angle as small as possible, weak zero drift still exist when the resistance is very large. Therefore it is necessary to further adjust zero drift with an auxiliary circuit at the input of the integrator.

\section{AC high-voltage measuring system characteristics}

\subsection{Steady state characteristics}

In the performance analysis of $\mathrm{AC}$ high- voltage measuring system, the current voltage conversion circuit and high voltage standard capacitor are considered as a whole, first, the signal generator (AFG 3252) produce $10 \mathrm{~V}$ sine voltage signal, then the linear $\mathrm{HV}$ amplifier (Trek Model 10/40A-HS) can be adjusted the rate to 1100 times, high voltage acts on the high voltage standard capacitor, The current goes into the current voltage converting circuit, the output voltage of the signal generator and the current voltage conversion circuit are respectively acquired by the oscilloscope (TDS 3052), to establish the structure diagram as shown in Figure 2.

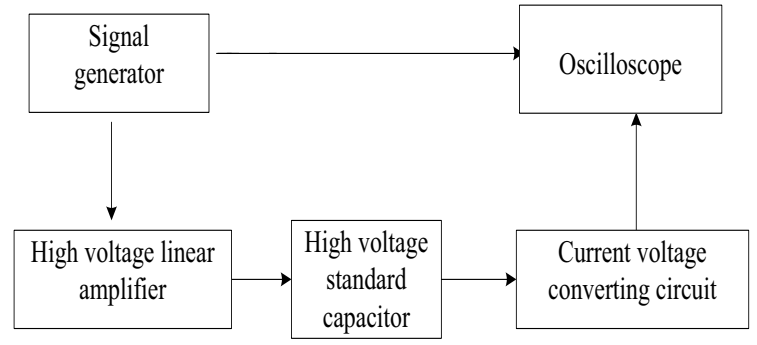

Figure 2. Test structure diagram of high voltage simulation

If high voltage signal is up, as shown in equation (4):

$$
u_{\mathrm{p}}=\sqrt{2} U_{\mathrm{P}} \sin \left(\omega_{0} t+\theta_{0}\right)
$$

Where $\mathrm{U}_{\mathrm{p}}$ is a part of the effective value of $\mathrm{AC}$ signal; $\omega_{0}$ for power system frequency;

$\theta_{0}$ for the initial phase of the voltage signal;

High voltage standard capacitor connected in parallel with the grid system, the capacitor current is IC, as shown in equation (5):

$$
I_{\mathrm{C}}(t)=C_{\mathrm{H}} \frac{\mathrm{d} u_{p}(t)}{\mathrm{d}(t)}=\sqrt{2} \omega_{0} C_{\mathrm{H}} U_{\mathrm{P}} \cos \left(\omega_{0} t+\theta_{0}\right)
$$

The current signal and primary voltage signal are differential relation with $90^{\circ}$ phase difference, It needs to be integrated to convert current signal into voltage signal, and then to make the same proportion and phase, The output voltage of the integrator goes into the inverter, as shown in equation (6):

$$
u_{\mathrm{q}}=\frac{\sqrt{2} C_{\mathrm{H}} U_{\mathrm{P}}}{R_{\mathrm{J}} C_{\mathrm{J}}} \sin \left(\omega_{0} t+\phi_{0}\right)=K u_{\mathrm{p}}
$$

$\mathrm{K}=\mathrm{C}_{\mathrm{H}} / \mathrm{R}_{\mathrm{J}} \mathrm{C}_{\mathrm{J}}$, The output voltage can be proportional to primary voltage signal amplitude with the same phase when the system is in steady state, and we can change the size of $\mathrm{K}$ according to the need of the regulation[5], steady state simulation experiments as follows.

(1) The wave forms of the output voltage $u_{q}$ and the input voltage $u_{p}$ in steady state are shown in Figure 3 . (Oscilloscope channel ch1 shows primary voltage, channel 2 shows for secondary output voltage).

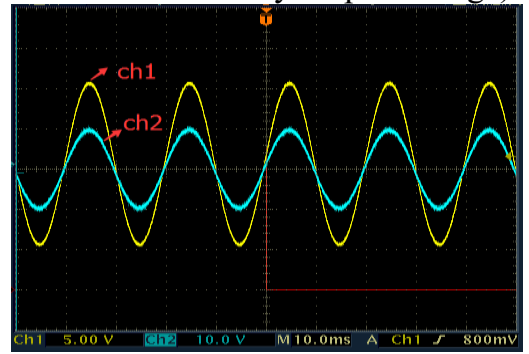

Figure 3. Primary and secondary voltage waveform 
(2) The input current and output voltage linearity curve, as shown in Figure 4.

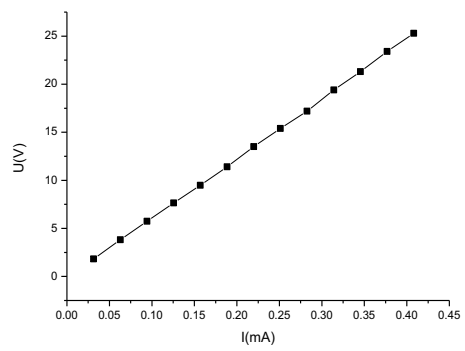

Figure 4. Input current and output voltage linearity curve

Through origin analysis of the fitting line, the sum of squared residuals is 0.11675 , the variance is 0.99982 , approximately equal to 1 . That is, the input current and output voltage has good linearity.

\subsection{Frequency characteristic}

The input and the output voltage waveform under different frequencies as shown in Figure 5.

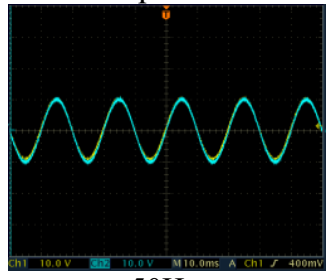

$50 \mathrm{~Hz}$

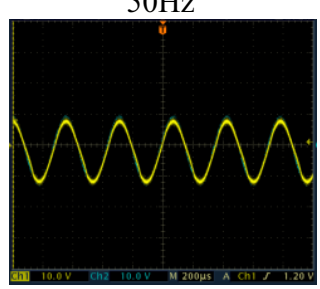

$2500 \mathrm{~Hz}$

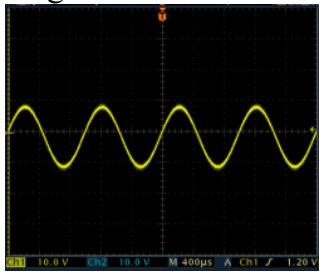

$1000 \mathrm{~Hz}$

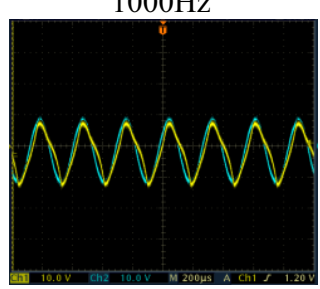

$3000 \mathrm{~Hz}$
Figure 5.Input and output voltage wave forms at different frequencies

The curve of the ratio error and phase error with frequency change shown in Figure 6.
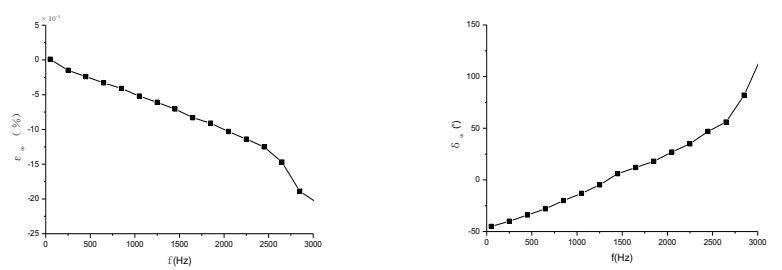

Figure 6. The ratio error and phase angle error curve with frequency

As can be seen from these wave forms, the AC highvoltage measuring system have good voltage output characteristic from $50 \mathrm{~Hz}$ to $2500 \mathrm{~Hz}$, at frequencies above $2500 \mathrm{~Hz}$, the output voltage waveform distortion occurs, as can be seen from Figure 6. Ranging from the low frequency to high frequency, the output voltage ratio error and phase error with frequency change in ideal range, it is found that the $\mathrm{AC}$ high-voltage measuring system has good frequency characteristics and output voltage stability.

\section{Error Analysis}

\subsection{The effect of temperature on AC HV measuring system}

Due to the temperature coefficient of high voltage standard capacitor is nearly zero; the effect of temperature variation on the current voltage conversion circuit is only analyzed here. The resistor or capacitor parameter of the current voltage conversion circuit relate to temperature, so the change of temperature affects the output of AC high-voltage measuring system.

If the input signal is sine signal, the output signal amplitude can be achieved by the above equation, as shown in equation (7):

$$
\left|u_{\mathrm{q}}\right|=\frac{C_{H}}{R_{\mathrm{J}} C_{\mathrm{J}}}\left|u_{\mathrm{p}}\right|=K\left|u_{\mathrm{p}}\right|
$$

Where $\mathrm{K}$ is related to the coefficient of resistance and capacitance. The size of $\mathrm{CH}$ is independent of temperature, So $\mathrm{K}$ is a coefficient associated with $\mathrm{RJ}$ and $\mathrm{CJ}$, as shown in equation (8):

$$
\frac{\mathrm{d} K}{K \mathrm{~d} T}=\frac{R_{J} C_{J}}{C_{H}} \cdot \frac{\mathrm{d}}{\mathrm{d} T}\left(\frac{C_{H}}{R_{J} C_{J}}\right)=-\left(\frac{\mathrm{d} R_{J}}{R_{J} \mathrm{~d} T}+\frac{\mathrm{d} C_{J}}{C_{J} \mathrm{~d} T}\right)
$$

Equation (8) shows that the temperature coefficient of $\mathrm{K}$ is the sum of the temperature coefficient of resistance and capacitance. If the temperature coefficient of resistance is $10^{-5} /{ }^{\circ} \mathrm{C}$, that of capacitance is $3 \times 10^{-5} /{ }^{\circ} \mathrm{C}$, and the change of temperature is $100^{\circ} \mathrm{C}$, then $(\mathrm{dK} / \mathrm{K})=0.4 \%$, so the temperature will be smaller impact on the $\mathrm{AC}$ high-voltage measuring system, the above temperature error can be further improved by selecting resistor or capacitor with low and equivalent temperature coefficient $[6,7]$.

\subsection{The Influence of the sundry capacitance and other factors on AC HV measuring system}

The measuring system is mainly affected by the sundry capacitance to ground and the sundry capacitance to the high voltage. the sundry capacitance to ground due to be grounded at both ends[8], it will not affect the high voltage standard capacitor, sundry capacitance to high voltage equivalently parallels with the high voltage standard capacitor, so the introduction of high voltage standard capacitor can eliminate the influence of sundry capacitance on the accuracy of the measuring system. In addition to the above factors, we still have to consider the error caused by the high frequency noise interference, the DC offset of the operational amplifier, and the change of the frequency of the power network, but these errors can take some measures to restrain in order that the total error range can be controlled within the technical requirements of high voltage measuring system. 


\section{Conclusion}

A system for measuring $\mathrm{AC}$ high voltage using high voltage standard capacitor has been developed, takes advantage of minimum temperature coefficient and minimal dielectric loss of high voltage standard capacitor, the influence of temperature and the sundry capacitance on the test accuracy can be eliminated in a large extent, the system has the advantages of simple structure, high insulation strength, good temperature characteristics, good steady-state output characteristics, frequency characteristics and so on. According to the high voltage capacitor model in the market, the system can be applied to multiple voltage levels; it can also be used to form a measuring device for extracting high voltage signal in the laboratory.

\section{Acknowledgment}

The authors would like to thank the State Grid Electric Power Research Institute in Jiangsu Province for their financial support.

\section{References}

1. Faifer M, Toscani S, Ottoboni R Electronic Combined Transformer for Power-Quality Measurements in HighVoltage Systems [J]. Instrumentation \& Measurement IEEE Transactions on (2011)

2. Kosakada M, Watanabe H, Ito $\mathrm{T}$, et al. Integrated substation systems-harmonizing primary equipment with control and protection systems[C] Transmission and Distribution Conference and Exhibition Asia Pacific. IEEE/PESIEEE, 2002:1020-1025 vol.2(2003)

3. Feng Y, Wang X Q, Bing A I, et al. Study on the Surrounding Influence of Electric Field on Accuracy of Electronic Voltage Transformer with Capacitive Voltage Divider [J]. Electrical Measurement \& Instrumentation(2014)

4. Kumada A, Hidaka K. Directly High-Voltage Measuring System Based on Pockels Effect[J]. Power Delivery IEEE Transactions on, 28(3):1306-1313(2013)

5. CEASE $\mathrm{T} \mathrm{W}$, JOHNSTON P A Magneto-optic Current Transducer[J]. IEEE Trans on Power Delivery(1990)

6. Bak J G, Lee S G, Team T K P, et al. Performance of the magnetic sensor and the integrator for the KSTAR magnetic diagnostics $[\mathrm{J}]$. Review of Scientific Instruments, 75(10):4305-4307(2004)

7. Donaldson E F, Gibson J R, Jones G R, et al. Hybrid optical current transformer with optical and power-line [J]. IEEE Proceedings-Generation, Transmission and Distribution(2000)

8. Chavez P P, Rahmatian F, Jaeger N A F. $230 \mathrm{kV}$ optical voltage transducer using a distributed optical electric field sensor system[C] Transmission and Distribution Conference and Exposition, $2001 \mathrm{IEEE} / \operatorname{PESIEEE(2001)~}$ 